\title{
EXAMINATION OF OLDER DRIVER STEERING ADAPTATION ON A HIGH PERFORMANCE DRIVING SIMULATOR
}

\author{
Daniel V. McGehee \\ John D. Lee \\ Matthew Rizzo \\ Kirk Bateman \\ Colleges of Engineering, Medicine and Public Policy Center \\ University of Iowa \\ Iowa City, Iowa, USA \\ E-Mail: daniel-mcgehee@uiowa.edu
}

\begin{abstract}
Summary: The objective of this study was to examine how long it takes for older drivers to adapt their steering control on a fixed-base driving simulator. We hypothesized that older drivers achieve maximum training benefit within the first few minutes of a driving simulation. Thirteen drivers over 65 years of age drove a four-channel, $150^{\circ}$ forward field-ofview, $50^{\circ}$ rear field-of-view, fixed-base driving simulator for 25 minutes. We used a six-degree steering wheel reversal criterion to evaluate drivers' adaptation to the simulator. Since drivers' adapt to a simulator over time, we examined the number of steering wheel reversals greater than six degrees that occurred per minute during each of three sections, the start, middle and end of the 25-minute drive. The results showed that older drivers needed about three minutes to adapt and get the "feel" of the simulator. Before this time driving behavior in the simulator may not be representative of actual driving performance. These results provide preliminary support for assuming that an adaptation period as short as five minutes may enable drivers to adapt to the driving simulator and drive normally.
\end{abstract}

\section{INTRODUCTION/OBJECTIVES}

A common question in experimental driving research is, "how long does it take for a research participant to adapt to the simulator?” Interactive driving simulators do not completely replicate the driving experience and require time for drivers to adapt to the vehicle dynamics and perceptual cues of the simulator. Older drivers may be particularly vulnerable to differences between the simulator and the real vehicle. Driving simulator researchers usually make subjective judgments as to a person's adaptation to a simulator. Most driving simulator studies have practice sessions lasting from 5-15 minutes, but specific objective measurements of adaptation and training to pre-determined criteria seem to be lacking.

\section{DRIVING SIMULATOR}

The Simulator for Interdisciplinary Research in Ergonomics and Neuroscience (SIREN) is a state-of-the-art driving simulator designed for research in the clinical setting. Located in the 
Neurology Department at the University of Iowa's College of Medicine, SIREN is comprised of a 1994 GM Saturn with specially designed electronic sensors and pinhole video cameras for recording driver performance. SIREN also includes a sound system and surrounding screens $\left(150^{\circ}\right.$ forward field of view, $50^{\circ}$ rear field of view), four LCD projectors with image generators and an integrated host computer, and another computer for scenario design, control and data collection.

\title{
METHODS
}

Thirteen drivers over 65 years of age drove the SIREN for 25 minutes. The drive was comprised of rural, two-lane roadways with traffic. Participants interacted with other vehicles, made judgments about traffic lights, and responded to potential collision conditions. From this drive, three one-mile (1.6 kilometer) sections of uneventful driving were extracted at the start, middle, and end of the drive to examine steering behavior. The first section of interest began at the start of the drive; subsequent sections started near the four kilometers and 15 kilometer points respectively.

\begin{abstract}
ANALYSIS
Steering wheel reversals and overall steering movement have been used in driving research to examine attention demand and vehicle control ability. A steering wheel reversal is operationally defined as a deflection of the steering wheel away from a central or neutral position (i.e., where the wheels are completely straight) followed by a reversal of the steering wheel back to the neutral position. Steering wheel reversals do not include steering movements associated with an intentional vehicle turning maneuver, such as curve negotiation. To differentiate the numerous small steering wheel reversals associated with normal driving from those associated with high levels of cognitive demand, a threshold of six degrees has been adopted. Steering reversals of more than six-degrees indicate impaired steering control associated with high attentional demands or poor control ability (Wierwille \& Gutmann, 1978). As the level of demand increases, drivers tend to make fewer, but larger, steering corrections or steering reversals. An increase in steering reversals greater than 6 degrees, with a decreased frequency of small reversals, has been associated in the laboratory and in on-the-road experiments with increased attentional demands. This standard has been used to measure the attentional demands on the driver for more than 20 years. . We used the six-degree steering wheel reversal criterion to evaluate drivers' adaptation to the simulator, with the large steering movements indicating a high cognitive load and impaired vehicle control associated with poor adaptation to the simulator. Since drivers' adapt to a simulator over time, we examined the number of steering wheel reversals greater than six degrees that occurred per minute during each of three sections of the 25-minute drive. In addition, we examined overall steering movement for each section defined as the mean absolute value of the steering wheel. Finally, we plotted the time series of the steering data by segment to visualize how frequently steering reversals exceeded the $+/-6$ degree steering envelope.
\end{abstract}




\section{RESULTS}

As Table 1 shows, there were significant decreases in the total number of steering wheel reversals per minute and overall variance in steering between the first segment relative to the second and third.

Table 1. Mean deviations greater than +/- 6 degrees per minute by road segment

\begin{tabular}{l|lll} 
& $\begin{array}{l}\text { Road Segment } \\
\mathbf{1}\end{array}$ & $\begin{array}{l}\text { Road Segment } \\
\mathbf{2}\end{array}$ & $\begin{array}{l}\text { Road Segment } \\
\mathbf{3}\end{array}$ \\
\hline $\begin{array}{l}\text { Mean } \\
\text { Deviations/Minute }\end{array}$ & $10.40($ SD 6.37) & $2.86($ SD 4.14) & 3.57 (SD 3.88) \\
\hline $\begin{array}{l}\text { Overall Steering } \\
\text { Movement }\end{array}$ & $9.82($ SD 8.51) & $2.20($ SD 0.99) & 2.63 (SD 1.50) \\
& & &
\end{tabular}

Inferential comparison of deviations per minute (Wilcoxon signed rank tests)

Segment 1 vs. $2 \mathrm{p}=0.003$

Segment 1 vs. $3 \mathrm{p}=0.008$

Segment 2 vs. $3 \mathrm{p}=0.333$

Inferential comparison of overall steering movement (Wilcoxon signed rank tests)

Segment 1 vs. $2 \mathrm{p}<0.001$

Segment 1 vs. $3 \mathrm{p}<0.001$

Segment 2 vs. $3 \mathrm{p}=0.463$

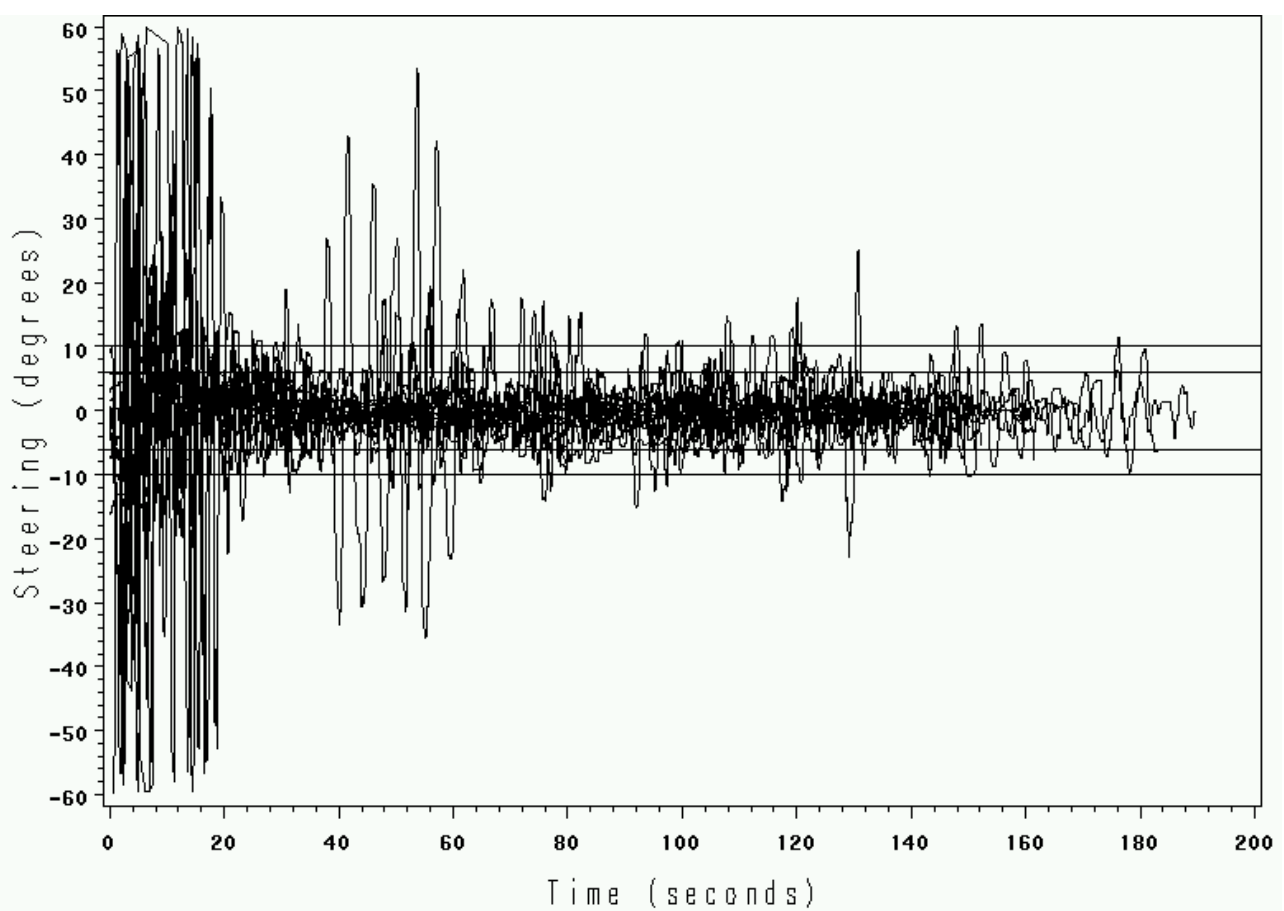

Figure 1. Time series of steering wheel position of all 13 drivers for the first segment. 
Since we were able to demonstrate significant differences between the segments, we then plotted the data of all 13 subjects to determine when or how long it took for the older drivers to stay within +/-6 degrees of steering. Figure 1 illustrates a time series of the steering behavior of the drivers in the first segment. Error bars depicting +/- 6 and 10 degrees have been placed on the plot. The plot shows that after approximately two minutes most drivers fall within the six-degree criteria, and after three minutes, almost all stay within the six-degree bounds. Figures 2 and 3 show the time series of the second and third segments later in the drive. Error bars depicting +/6 and 10 degrees have been placed on these plots. While there is one subject that exceeded the criteria, note that the drivers all nearly fall within the +/-6 and 10 degree criteria.

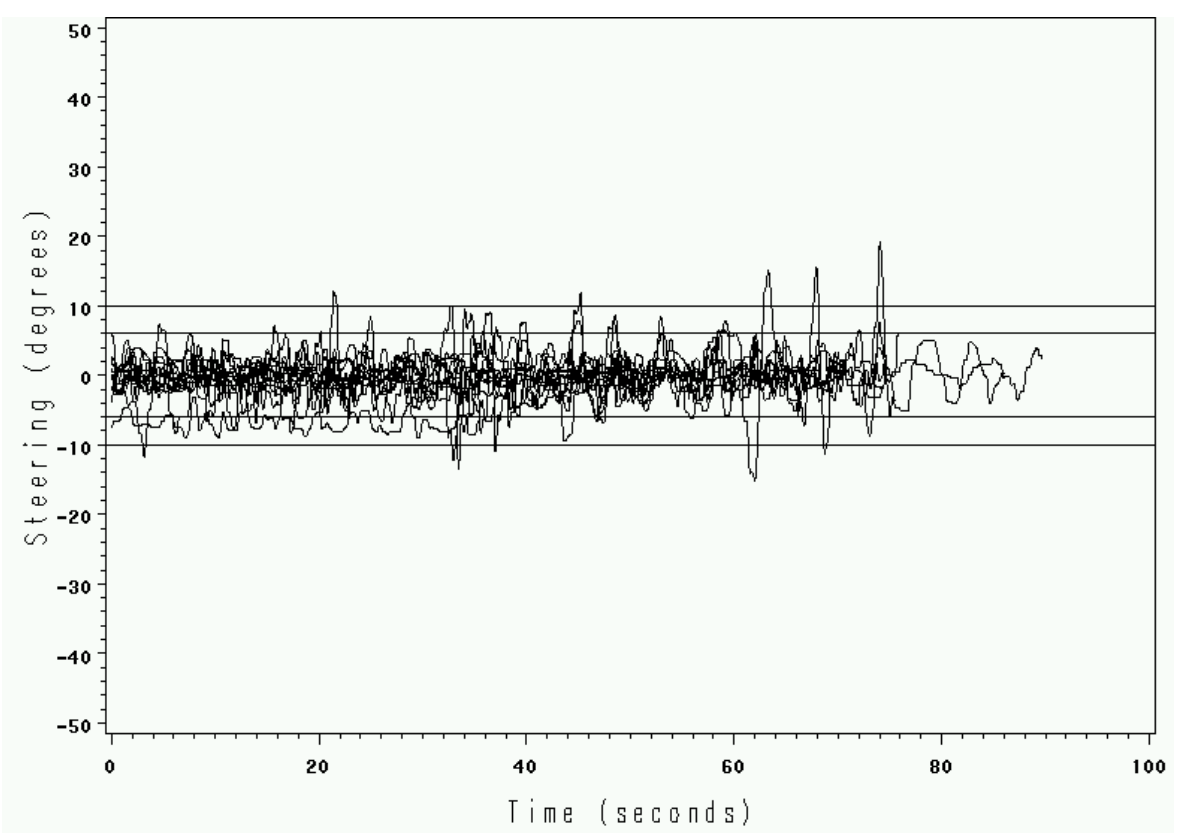

Figure 2. Time series of steering wheel position of all 13 drivers for the second segment.

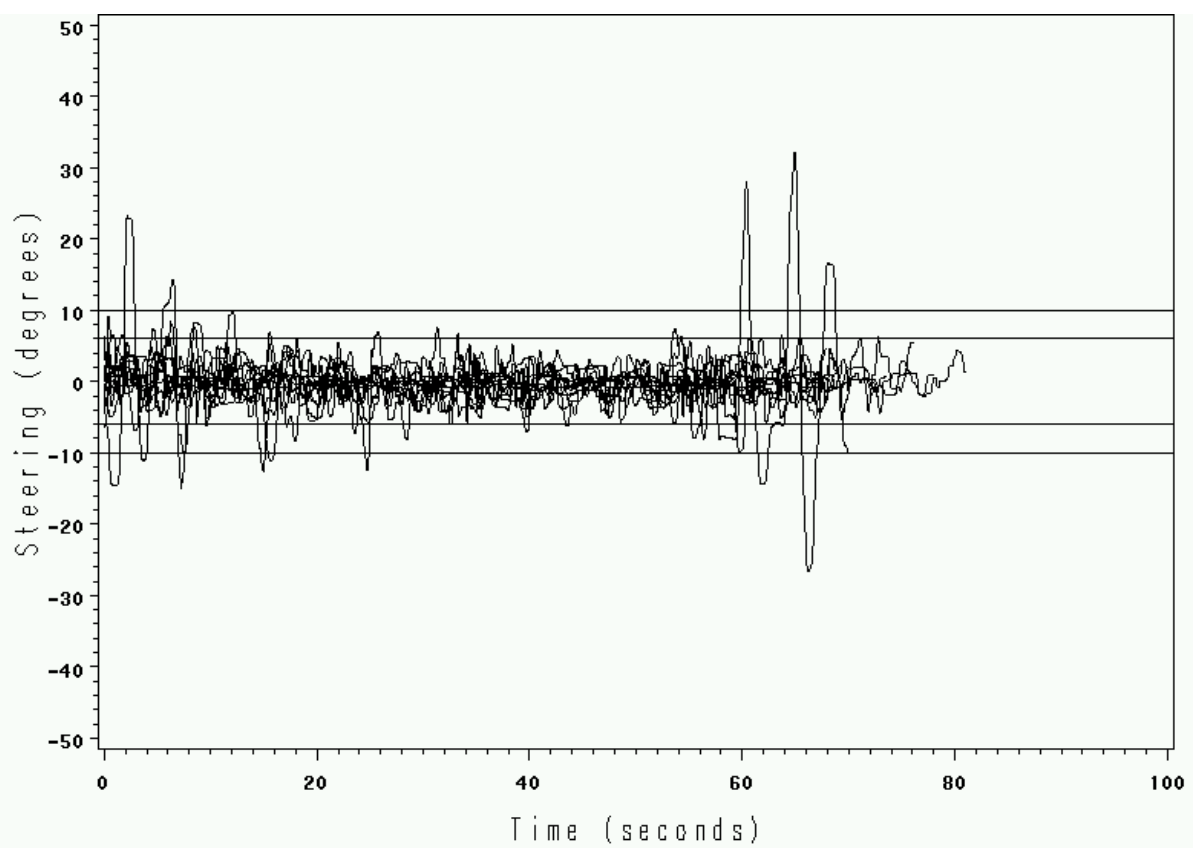

Figure 3. Time series of steering wheel position of all 13 drivers for the third segment. 


\section{CONCLUSIONS}

Based on these data, we can empirically show that older drivers need about three minutes to adapt and get the "feel" of the simulator. Before this time driving behavior in the simulator may not be representative of actual driving performance. Future analyses will examine other driving performance metrics to develop general criteria for determining practice requirements. These results provide preliminary support for assuming that an adaptation period as short as five minutes may enable drivers to adapt to the driving simulator and drive normally.

\section{ACKNOWLEDGEMENTS}

This research was supported by the U.S. National Institutes of Aging (NIA AG15071 \& NIA AG17177). The authors would also like to thank Julie Jermeland, a research engineer at the SIREN laboratory for setting up this experiment and collecting these data.

\section{REFERENCES}

W.W. Wierwille, and F. Gutmann. (1978). “Comparison of primary and secondary task measures as a function of simulated vehicle dynamics and driving conditions.” Human Factors, 20 (2), 233-244. 